\title{
Reviewers of the Journal of Artificial Organs for Volume 16
}

(c) The Japanese Society for Artificial Organs 2013

The following list of referees covers volume 16 of 2013:

\author{
Abe, Takaya \\ Akiyama, Masatoshi \\ Akutsu, Toshinosuke \\ Anai, Hirofumi \\ Ando, Ryoichi \\ Date, Hiroshi \\ Endo, Yoshihiro \\ Fujimoto, Keiji \\ Fujisato, Toshiya \\ Fujita, Tomoyuki \\ Fukamachi, Kiyotaka \\ Gojo, Satoshi \\ Hanazaki, Kazuhiro \\ Haraguchi, Yoshikura \\ Hashimoto, Kazuhiro \\ Hashimoto, Shigehiro \\ Hashimoto, Yoshiya \\ Higuchi, Chieko \\ Hiramatsu, Yuji \\ Hiroyuki, Kuromitsu \\ Homma, Akihiko \\ Horiuchi, Takashi \\ Hoshiba, Takashi \\ Hoshino, Masami \\ Ichiba, Shingo \\ Ichikawa, Hajime \\ Ichioka, Shigeru \\ Iguchi, Atsushi \\ Inoue, Hajime \\ Ishikawa, Toshiyuki \\ Itoh, Hideshi \\ Iwasaki, Kiyotaka \\ Iwata, Hiroo \\ Izumi, Yuichi
}

$\begin{array}{ll}\text { Kakibuchi, Masao } & \text { Morimoto, Naoki } \\ \text { Kakinoki, Sachiro } & \text { Morita, Shigeki } \\ \text { Kanamori, Toshiyuki } & \text { Moro, Toru } \\ \text { Kawaharada, Nobuyoshi } & \text { Nagaki, Masahito } \\ \text { Kawahito, Koji } & \text { Nakamura, Makoto } \\ \text { Kawakami, Hiroyoshi } & \text { Nakamura, Takao } \\ \text { Kawanishi, Hideki } & \text { Nakano, Kiyoharu } \\ \text { Kimura, Tsuyoshi } & \text { Nakayama, Yasuhide } \\ \text { Kinugasa, Eriko } & \text { Nam, Kwangwoo } \\ \text { Kishi, Kazuo } & \text { Nishida, Kenro } \\ \text { Kobayashi, Hisatoshi } & \text { Nishida, Masahiro } \\ \text { Komatsu, Kanshi } & \text { Nunoda, Shinichi } \\ \text { Kosaka, Ryo } & \text { Oda, Shigeto } \\ \text { Kukita, Kazutaka } & \text { Ohata, Toshihiro } \\ \text { Kuroyanagi, Yoshimitsu } & \text { Ohtsuki, Chikara } \\ \text { Mahara, Atsushi } & \text { Ohura, Norihiko } \\ \text { Maruyama, Osamu } & \text { Okazaki, Masayuki } \\ \text { Masai, Takafumi } & \text { Ono, Minoru } \\ \text { Masaki, Aihara } & \text { Ono, Tsutomu } \\ \text { Masuda, Munetaka } & \text { Onodera, Hisashi } \\ \text { Matsusaki, Michiya } & \text { Ozaki, Shigeyuki } \\ \text { Matsushita, Michiaki } & \text { Saiki, Yoshikatsu } \\ \text { Matsushita, Shonosuke } & \text { Saito, Shunsuke } \\ \text { Mineshima, Michio } & \text { Sakaguchi, Taichi } \\ \text { Mitsumaru, Atsuhiro } & \text { Sakakibara, Shunsuke } \\ \text { Mitsuno, Masataka } & \text { Sakiyama, Ryoichi } \\ \text { Miyamoto, Keiichi } & \text { Sase, Kazuhiro } \\ \text { Miyamoto, Shinji } & \text { Sato, Koichi } \\ \text { Miyasaka, Takehiro } & \text { Sato, Yuichi } \\ \text { Miyazawa, Mitsuo } & \text { Sezai, Akira } \\ \text { Mizuno, Hiroshi } & \text { Shiga, Hidetoshi } \\ \text { Mizuno, Toshihide } & \text { Shiiya, Norihiko } \\ \text { Mochizuki, Seiichi } & \\ \text { Momose, Naoki } & \end{array}$

\author{
Shiraishi, Yasuyuki \\ Sueda, Taijiro \\ Suzuki, Shigehiko \\ Tabata, Yasuhiko \\ Tajikawa, Tsutomu \\ Takami, Yoshiyuki \\ Takano, Hiroshi \\ Takewa, Yoshiaki \\ Tanaka, Akira \\ Tanaka, Masaru \\ Tanaka, Rica \\ Tani, Tohru \\ Tanihara, Masao \\ Tanoue, Yoshihisa \\ Tayama, Eiki \\ Terashi, Hiroto \\ Toda, Koichi \\ Todo, Mitsugu \\ Tomita, Naohide \\ Topley, Nicholas \\ Usui, Akihiko \\ Yagi, Takanobu \\ Yamaji, Ken \\ Yamamoto, Kenichiro \\ Yamamoto, Masaya \\ Yamane, Takashi \\ Yamashita, Akihiro C. \\ Yamazaki, Kenji \\ Yanai, Mitsuru \\ Yano, Tetsuya \\ Yonekawa, Motoki \\ Yoshimi, Yasuo \\ Yoshizawa, Makoto
}

